\title{
EMBEDDINGS OF SURFACES IN EUCLIDEAN THREE-SPACE
}

\author{
BY C. E. BURGESS ${ }^{1}$
}

1. Introduction. We shall discuss problems of the following three general types, where $S$ is a 2 -sphere in Euclidean three dimensional space $E^{3}$. (We use "2-sphere" to mean any homeomorphic image of the round sphere described by $x^{2}+y^{2}+z^{2}=1$.)

1.1. Problem. Identify conditions under which there is a homeomorphism $h$ of $E^{3}$ onto itself such that $h(S)$ is the round sphere described by $x^{2}+y^{2}+z^{2}=1$.

1.2. Problem. Identify conditions under which there is a homeomorphism $h$ of $E^{3}$ onto itself such that $h(S \cup \operatorname{Int} S)$ is the round ball described by $x^{2}+y^{2}+z^{2} \leqq 1$. (We use "Int $S$ " to denote the bounded component of $E^{3}-S$.)

1.3. Problem. For a closed subset $X$ of $S$, identify conditions under which there is a homeomorphism $h$ of $E^{3}$ onto itself such that $h(X)$ is a subset of the round sphere $x^{2}+y^{2}+z^{2}=1$.

We use $B^{3}$ to denote the round ball described by $x^{2}+y^{2}+z^{2} \leqq 1$, and we call any set homeomorphic with $B^{3}$ a 3-cell. We define a crumpled cube to be any set homeomorphic to $S \cup \operatorname{Int} S$, where $S$ is a 2 -sphere in $E^{3}$. We define a 2-sphere $S$ in $E^{3}$ to be tame if there is a homeomorphism of $E^{3}$ onto itself which carries $S$ onto the round sphere $x^{2}+y^{2}+z^{2}=1$. Any 2 -sphere in $E^{3}$ without this property is said to be wild. We say that a closed subset $X$ of a 2 -sphere in $E^{3}$ is tame if $X$ is a subset of some tame 2-sphere. With these definitions, the three problems above can be stated as follows.

1.1'. Problem. Identify conditions under which a 2 -sphere in $E^{3}$ is tame.

$1.2^{\prime}$. Problem. Identify conditions under which a crumpled cube is a 3-cell.

1.3'. Problem. Identify conditions under which a closed subset of a 2-sphere in $E^{3}$ is tame.

Similar questions about 1 -spheres, or simple closed curves, in the Euclidean plane $E^{2}$ were answered by Schoenflies [92] about seventy years ago. The answers were simpler for $E^{2}$ than for $E^{3}$, however, as all 1 -spheres are tame in $E^{2}$. Thus every 1 -sphere in $E^{2}$ is the boundary of a 2-cell, or disk, and every closed subset of a 1 -sphere in $E^{2}$ is tame.

An address delivered to the American Mathematical Society at the University of Southern California on November 23, 1974, by invitation of the Committee to Select Hour Speakers for Far Western Sectional Meetings; received by the editors January 20, 1975.

AMS (MOS) subject classifications (1970). Primary 55A30, 55A35, 57A10, 57A35, 57A45, 57A50, 57C55.

Key words and phrases. Tame spheres, wild spheres, tame subsets of spheres, taming sets, crumpled cubes.

${ }^{1}$ Research partially supported by NSF-GP-20292. 


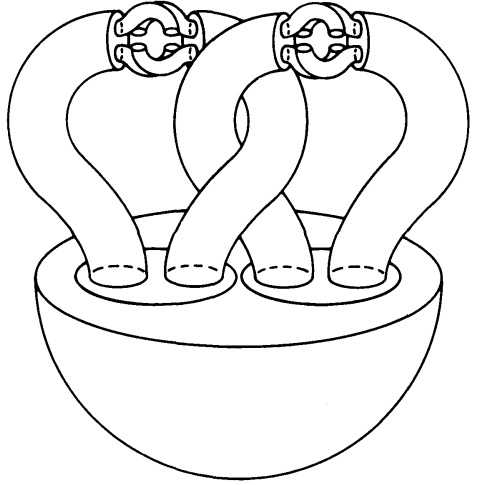

FIGURE 1

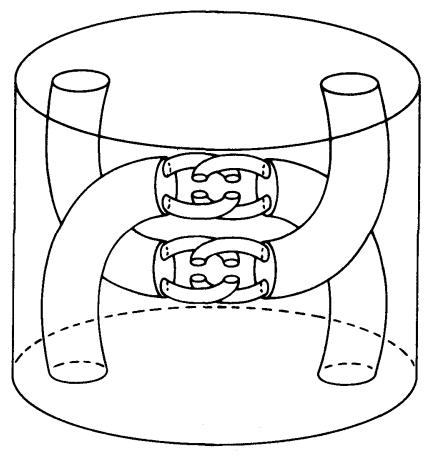

Figure 2

For 2-spheres in $E^{3}$, Problems 1.1 and 1.2 were first seriously pursued in the early 1920 's by Alexander. He indicated a proof to show that every polyhedral 2-sphere in $E^{3}$ is tame [1], and he announced, erroneously, that every 2 -sphere in $E^{3}$ is tame [2, p. 10]. However, he then corrected his own announcement by describing two types of wild 2-spheres in $E^{3}$. For the first example, he noticed that a wild Cantor set in $E^{3}$, known as Antoine's necklace [5], [6], could be described as a subset of a 2 -sphere in $E^{3}$, and thus he identified a 2-sphere which is wild on a wild Cantor set [3]. He then described a wild 2-sphere which is wild on a tame Cantor set [2]. This latter example, known now as the "Alexander horned sphere," is indicated in Figure 1. We indicate that this sphere is wild by observing that one of the components of its complement is not simply connected. This has been done precisely by Bing [15].

The 2-sphere $S$ in Figure 1 is the boundary of a 3-cell in $E^{3}$, but $S \cup E x t S$ is not homeomorphic with the closure of $E^{3}-B^{3}$; i.e., $S$ is tame from Int $S$ but

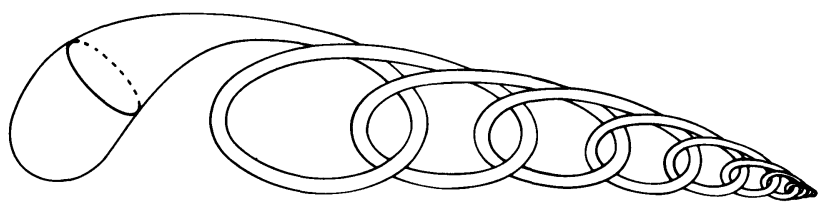

FIGURE 3

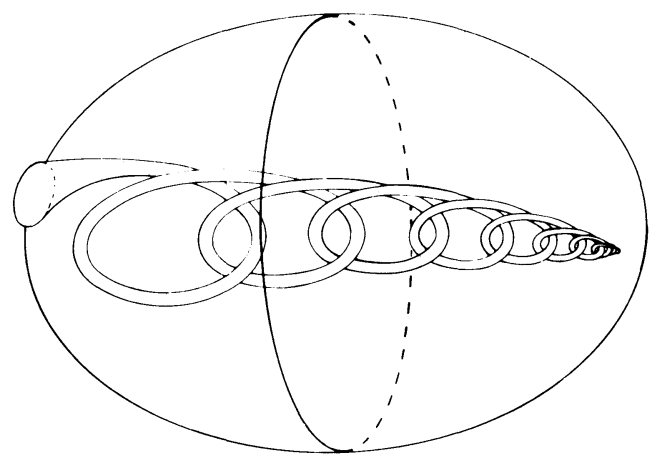

FIGURE 4 
not from Ext $S$. However, in Figure 2, $S$ is described so that $S \cup I n t S$ is not a 3 -cell and $S$ is tame from Ext $S$. We suggest that this crumpled cube might be called "Alexander's bowling ball."

No further significant progress on Problems 1.1, 1.2, and 1.3 occurred until 1948 when Fox and Artin [67] described a wild 2-sphere $S$ in $E^{3}$ such that $S$ is locally tame except at one point. Spheres of this type are indicated in Figures 3 and 4, where $S \cup I n t S$ is a 3-cell in Figure 3 but not in Figure 4 .

Further progress on problems about tame embeddings in $E^{3}$, and in 3-manifolds, resulted from work by Moise and Bing in the early 1950's. Moise [88] first showed that every 3-manifold can be triangulated, and shortly thereafter Bing [11] and Moise [89] proved, independently, that locally tame compact sets in 3-manifolds are tame. Thus a 2-sphere in $E^{3}$ is tame if it is locally tame, and this could be considered a generalization of Alexander's earlier theorem that polyhedral 2-spheres in $E^{3}$ are tame. Bing's alternative proof that 3-manifolds can be triangulated [14] was based upon his proof, in 1955 , that every 2 -sphere in $E^{3}$ can be homeomorphically approximated with a polyhedral sphere [12]. In an invited address to the Society in December 1956 [70], Harrold discussed what was then known about locally tame embeddings of curves and surfaces in 3-manifolds. However, some of the main tools for studying embeddings of surfaces in 3-manifolds were not available until after he presented his paper. These major developments were proofs of Dehn's Lemma, the Sphere Theorem, and the Loop Theorem, which were all presented by Papakyriakopoulos in the latter part of 1956 [90], [91], and Bing's proof of the Side Approximation Theorem for 2-spheres which appeared in 1963 [19].

My own interest in tameness criteria for 2-spheres, and subsets of them, began in the early 1960's, and it was my privilege, during the 1960 's, to be associated with several graduate students who studied this topic in their theses and who continued similar work into their postdoctoral years. Some important contributions to its development have been made by J. W. Cannon, W. T. Eaton, F. W. Lister, and L. D. Loveland. In 1971, J. W. Cannon and I jointly wrote an extensive survey of results on the development of this topic [32]. It will probably be apparent to anyone who seriously reviews what has been done on this topic that it would be more appropriate for James Cannon to be presenting an address summarizing this work.

We shall attempt to present an emphasis of this topic which is slightly different from the one in [32] and, at the same time, less extensive. However, some overlapping will be necessary, and we shall rely on [32] for some definitions and for a more extensive bibliography. We do not include properties of piercing points of 2-spheres and crumpled cubes, but a summary is included in [32, pp. 292-295]. An outline of a suggested procedure for studying embeddings of surfaces in $E^{3}$ is included in [32, pp. 261-263]. We would now supplement this suggested outline by adding Cannon's work on ULC properties of neighborhoods of surfaces in $E^{3}[40]$, his alternative proofs of Bing's approximation theorems [41], and his tameness criteria for closed subsets of 2-spheres in $E^{3}$ [39]. Our main attention will be, in $\S 4$, on 
conditions which imply that a crumpled cube is a 3-cell. Then brief indications will be given, in $\$ \S 5,6$, and 7 , of similar criteria for tameness of 2-spheres, and subsets of them, and of taming sets for 2-spheres and crumpled cubes. In $\S 8$, we will indicate extensions of results about 2-spheres in $E^{3}$ to 2-manifolds in 3-manifolds. Various types of wild 2-spheres will be mentioned in $\$ 9$, and some brief comments about codimension one spheres in $E^{n}$ will be included in $\$ 10$. For the brief summary, in $\$ 10$, of higher dimensional work, I greatly appreciate several conversations with R. J. Daverman, as $\$ 10$ is based substantially on his recent work which he has summarized in an expository paper [56].

2. Building disks and spheres from singular ones. As indicated above in the introduction, three very important theorems, known as Dehn's Lemma, the Sphere Theorem, and the Loop Theorem, were first proved by Papakyriakopoulos in 1956 [90]. Some alternative proofs, and some slight variations in the theorems, have subsequently appeared in [73], [91], [94], [99], [100], [101], [40, 3.6], [32, Theorem 4.5.4]. For our purposes in this paper, these three theorems are stated in the following geometric forms.

2.1. Dehn's Lemma. If $f$ is a piecewise linear map of a polyhedral disk $D$ into a triangulated 3-manifold $M$ and $U$ is a neighborhood in $M$ of the singularities $^{2}$ of $f$ such that $U \cap f(\mathrm{Bd} D)=\varnothing$, then there is a piecewise linear homeomorphism $f^{\prime}: D \rightarrow f(D) \cup U$ such that $f^{\prime}|\operatorname{Bd} D=f| \operatorname{Bd} D$.

2.2. Sphere Theorem. If $M$ is an orientable triangulated 3-manifold and $f$ is a map of a 2-sphere $S$ into an open subset $U$ of $M$ such that $f$ cannot be shrunk to a point in $U$, then there is a polyhedral 2-sphere $S^{\prime}$ in $U$ that is not nullhomotopic in $U$.

2.3. Loop THEOREM. If $M$ is a 3-manifold-with-boundary, $K$ is a component of $\mathrm{Bd} M$, and $L$ is a loop in $K$ that is nullhomotopic in $M$ but not in $K$, then there is a disk $D$ such that $\mathrm{Bd} D \subset K$, Int $D \subset \operatorname{Int} M$, and $\mathrm{Bd} D$ is not the boundary of a disk in $K$.

3. Polyhedral approximations of surfaces. Bing first presented, in 1955, a proof that every 2 -sphere in $E^{3}$ can be homeomorphically approximated with a polyhedral sphere [12], and he then used an adaptation of this theorem for open subsets of 2-spheres in his alternative proof that 3-manifolds can be triangulated [14].

3.1. Approximation Theorem. If $S$ is a 2 -sphere in $E^{3}$ and $\varepsilon>0$, then there is a homeomorphism $h: S \rightarrow E^{3}$ such that $h(S)$ is polyhedral and $h$ moves no point more than a distance $\varepsilon$.

This theorem and Theorem 4.1.1 were used by Bing to prove that there do not exist uncountably many disjoint wild 2-spheres in $E^{3}$ [32, Theorem 3.6.1]. While there do exist uncountably many disjoint wild disks in $E^{3}$ [98], all

\footnotetext{
${ }^{2}$ We define the singularities of $f$ to be the closure of $\left\{x \mid x \in f(D)\right.$ and $f^{-1}(x)$ is nondegenerate $\}$.
} 
except countably many disks in such a collection would be locally tame except on their boundaries and hence would be subsets of 2-spheres [85].

A much more useful approximation theorem for 2-spheres in $E^{3}$, known as the Side Approximation Theorem, was first proved by Bing about 1960 [19], and an alternative proof was presented by Cannon about 1970 [41].

3.2. Side Approximation TheOREM. If $S$ is a 2-sphere in $E^{3}$ and $\varepsilon>0$ then there exist a homeomorphism $h: S \rightarrow E^{3}$ and a finite set of $\varepsilon$-disks $E_{1}$, $E_{2}, \cdots, E_{n}$ in $h(S)$ such that:

(1) $h$ moves no point more than a distance $\varepsilon$;

(2) $h(S)$ is polyhedral;

(3) $h(S)-\bigcup_{i=1}^{n}$ Int $E_{i} \subset$ Int $S$.

This theorem can be combined with properties of $E^{2}$ to obtain the following additional requirement:

(4) There exists a finite set of disjoint $\varepsilon$-disks $D_{1}, D_{2}, \cdots, D_{m}$ in $S$ such that $S-\bigcup_{i=1}^{m}$ Int $D_{i} \subset \operatorname{Ext} h(S)$.

A similar theorem can be obtained where (3) and (4) are changed to:

(3') $h(S)-\bigcup_{i=1}^{n}$ Int $E_{i} \subset$ Ext $S$;

(4') $S-\bigcup_{i=1}^{m}$ Int $D_{i} \subset$ Int $h(S)$.

The proofs by Bing and Cannon can be briefly contrasted as follows. The main part of Bing's proof involved simplifying the intersection of a 2-sphere with the 1-simplexes and 2-simplexes of a triangulation of $E^{3}$. His proof was mainly based on properties of $E^{2}$ and some simple linking theory for $E^{3}$. Cannon established the existence of a triangulation $T$ for a 2 -sphere $S$ in $E^{3}$ such that the 1-skeleton of $T$ has a tubular neighborhood in $E^{3}$. Then he used Dehn's Lemma and the Sphere Theorem to obtain a polyhedral 2-sphere from the boundary of this tubular neighborhood and from singular disks near the 2-simplexes of $T$.

A third proof of Theorem 3.2 can be obtained by combining work by Bing and Cannon. Bing had noticed [22, p. 155] that the general case in his proof could be simplified provided the following theorem could be proved independently of the Side Approximation Theorem.

3.3. THEOREM. If $L$ is a straight line and $S$ is a 2-sphere in $E^{3}$, then for each $\varepsilon>0$ there is a homeomorphism $h$ of $E^{3}$ onto itself such that $L \cap h(S)$ is finite and $h$ is the identity homeomorphism except in an $\varepsilon$-neighborhood of $S \cap L$.

Bing obtained the first proof of this theorem, but his proof depended upon the Side Approximation Theorem [22]. Recently, Cannon obtained a short proof that does not depend upon the Side Approximation Theorem [43]. Both proofs depended upon the existence of small meshed triangulations of $S$ with tame 1-skeletons, and Cannon used some of his work in [40] to obtain such a triangulation without using the Side Approximation Theorem. Bing [17] had earlier obtained such triangulations as a consequence of the Side Approximation Theorem. 
In [19], Bing included a Side Approximation Theorem for open subsets of 2-spheres in $E^{3}$. A few years later, in [21], he indicated how to use some of the consequences of the Side Approximation Theorem to obtain a simpler proof, and he advised the reader [21, p. 511] to ignore the proof in [19] and substitute the one in [21]. The theorem is valid for an open subset of a 2-manifold which separates a 3-manifold. Cannon's alternative proof, which is local in nature, can be used essentially without change to obtain this extended version of the Side Approximation Theorem.

Lister [79] improved Bing's version of the Side Approximation Theorem by requiring, in the conclusion of Theorem 3.2, that the $E_{i}$ 's intersect the $D_{i}$ 's in a one-to-one manner. He first used Dehn's Lemma to adjust the $E_{i}$ 's so that each $E_{i}$ intersected only one $D_{j}$. However this still permitted many $E_{i}$ 's to intersect the same $D_{i}$, but he then used properties of $E^{2}$ to amalgamate the $E_{i}$ 's so that each $D_{j}$ intersected only one $E_{i}$. Furthermore, he showed that the approximating homeomorphism $h$ could be adjusted so that, for each $i, h\left(D_{i}\right)=E_{i}$. His improved form of the theorem can be stated as follows as a two sided approximation theorem. (We use " $\varepsilon$-disk" to denote a disk of diameter less than $\varepsilon$ and " $\varepsilon$-homeomorphism" to denote a homeomorphism which moves no point more than a distance $\varepsilon$.)

3.4. Improved Side Approximation Theorem. If $S$ is a 2-sphere in $E^{3}$ and $\varepsilon>0$, then there exist a finite collection of disjoint $\varepsilon$-disks $D_{1}, D_{2}, \cdots, D_{n}$ in $S$ and two $\varepsilon$-homeomorphisms $\mathrm{g}, h: S \rightarrow E^{3}$ such that:

(1) $g(S)$ and $h(S)$ are disjoint polyhedral spheres;

(2) $\mathrm{g}\left(S-\bigcup_{i=1}^{n}\right.$ Int $\left.D_{i}\right) \subset$ Int $S$;

(3) $h\left(S-\bigcup_{i=1}^{n}\right.$ Int $\left.D_{i}\right) \subset$ Ext $S$;

(4) $S-\bigcup_{i=1}^{n}$ Int $D_{i} \subset$ Ext $g(S) \cap \operatorname{Int} h(S)$;

(5) for each $i,\left(g\left(D_{i}\right) \cap S\right) \cup\left(h\left(D_{i}\right) \cap S\right) \subset \operatorname{Int} D_{i}$.

This theorem has been useful in simplifying the proofs of some theorems which depended upon the Side Approximation Theorem and in proving some additional theorems. Some interesting illustrations of this are Daverman's alternative proof that any crumpled cube $C$ can be embedded in $E^{3}$ so that Bd $C$ is tame from $E^{3}-C$ [48], Lister's proof that tameness is preserved for certain subsets of $\mathrm{BdC}$ under such an embedding [80], and Cannon's simplified proof of Theorem 4.2.1 [35], [32, pp. 303-304].

Eaton [64] extended Lister's two sided approximation theorem (Theorem 3.4) so that each $D_{i} \cap\left(g\left(D_{i}\right) \cup h\left(D_{i}\right)\right)$ is a subset of the union of a finite number of disjoint disks in Int $D_{i}$ such that no one of them intersects both $g(S)$ and $h(S)$. He then used this theorem to develop a very useful criterion to determine whether certain types of cellular decompositions of $E^{3}$ yield $E^{3}$ [63], [65].

We can combine the forms of the Side Approximation Theorem proved by Bing, Cannon, Lister, and Eaton into the following general form of a two sided approximation theorem for open subsets of 2-manifolds in 3manifolds. 
3.5. TWO SIDED APPROXIMATION THEOREM FOR OPEN SUBSETS OF 2MANIFOLDS. If $M$ is a connected 2-manifold which separates a triangulated connected 3-manifold $M^{3}, U$ is an open subset of $M, f$ is a positive continuous real function defined on $U$, and $V$ and $W$ are the components of $M^{3}-M$, then there exist a null sequence $\left\{D_{i}\right\}$ of disjoint disks in $U$ and two homeomorphisms $\mathrm{g}, \mathrm{h}: U \rightarrow M^{3}$ such that:

(1) $\left\{D_{i}\right\}$ is locally finite in $U$;

(2) $g(U)$ and $h(U)$ are disjoint and locally polyhedral;

(3) for each $i$, Diam $D_{i}<f(x)$ for some $x \in D_{i}$;

(4) for each $x \in U, \rho(x, g(x) \cup h(x))<f(x)$;

(5) $g\left(U-\bigcup_{i=1}^{\infty}\right.$ Int $\left.D_{2 i-1}\right) \subset V$ and $h\left(U-\bigcup_{i=1}^{\infty}\right.$ Int $\left.D_{2 i}\right) \subset W$;

(6) for each i, $M \cap g\left(D_{2 i-1}\right) \subset$ Int $D_{2 i-1}$;

(7) for each $i, M \cap h\left(D_{2 i}\right) \subset \operatorname{Int} D_{2 i}$.

Some side approximations for a 2 -sphere $S$ in $E^{3}$ have been studied by Eaton [60] and Cannon [42] where the approximating sphere does not intersect both components of $E^{3}-S$ or where it is singular.

4. Criteria for a crumpled cube to be a 3-cell. If $S$ is a 2 -sphere in $E^{3}$, a crumpled cube is defined to be a space which is homeomorphic with $S \cup I n t S$. Where $C$ is such a crumpled cube and $h$ is a homeomorphism of $S \cup I n t S$ onto $C$, we use Bd $C$ and Int $C$ to denote $h(S)$ and $h($ Int $S)$, respectively. We discuss in this section some conditions which imply that a crumpled cube is a 3 -cell. These might be considered conditions which imply that a 2 -sphere in $E^{3}$ is tame from one side, and most of the conditions can be adapted to 2-sided tameness to give criteria for a 2-sphere to be tame in $E^{3}$. These related tameness criteria for a 2-sphere are discussed briefly in $\$ 5$. Some of the criteria we discuss are special cases of others, and some of them include positional requirements which are not possessed by every tame 3-cell in $E^{3}$. However, we can consider all of them to be criteria for a crumpled cube to be a 3-cell if, where necessary, we include in the hypothesis the additional requirement that the given crumpled cube be homeomorphic with one which satisfies the positional requirements.

A study of the tameness criteria discussed in this section and in $\$ 5$ is based mainly on Dehn's Lemma and the Side Approximation Theorem for 2spheres. The Sphere Theorem and the Loop Theorem are used in the development of some of the criteria, and Cannon's work on taming sets and $*$-taming sets, discussed in $\$ 7$, is essential in some of them.

4.1. HOMEOMORPHIC APPROXIMATIONS AND CONVERGING SEQUENCES OF SPHERES. For a crumpled cube $C$, we say that $\mathrm{Bd} C$ can be homeomorphically approximated in Int $C$ if for each $\varepsilon>0$ there exists a homeomorphism $h: B d C \rightarrow$ Int $C$ such that $h$ moves no point more than a distance $\varepsilon$.

4.1.1. THEOREM. A crumpled cube is a 3-cell if $\mathrm{Bd} \mathrm{C}$ can be homeomorphically approximated in Int $C$.

This theorem has been fundamental in the development of nearly all of the criteria discussed in $\$ \S 4$ and 5. It was first proved by Bing [13], with a rather 
complicated structure of "fences," before the Side Approximation Theorem for open subsets of 2-spheres was available. Recently, Cannon [41] has used Theorem 3.5, simplified to a one sided version for 2-spheres, to obtain a much simpler proof where Bing's fences are replaced with collars on 1-skeletons of triangulations of Bd C. (See $\$ 4.4$ for a definition of a collar.) Briefly, Cannon's proof can be outlined as follows. The hypothesis and Theorem 3.1 are used to obtain a sequence $\left\{S_{i}\right\}$ of disjoint polyhedral 2-spheres in Int $C$ converging homeomorphically to $\mathrm{Bd} \mathrm{C}$. (A definition of homeomorphic convergence can be found below with Theorem 4.1.3.) The existence of the sequence $\left\{S_{i}\right\}$ is combined with Theorem 3.5, the Side Approximation Theorem for open subsets of spheres, to obtain a collar for the 1-skeleton of a triangulation $T$ of $B d C$ and to extend this collar to collars of 1 -skeletons of successive subdivisions of $T$. These collars, along with the $S_{i}$ 's are used to build a network of polyhedral 2-spheres in Int $C$ which enable us to build and extend homeomorphisms with a similar network in a 3-cell. Details of this procedure are included in Cannon's alternative proof of Theorem 4.1.1 [41].

It is an unsolved problem to determine whether $C$ is a 3-cell if the homeomorphism $h$, in Theorem 4.1.1, is replaced with a map $f: B d C \rightarrow \operatorname{Int} C$ which moves no point more than a distance $\varepsilon$. Where such a map exists, Wilder [104] has defined Bd $C$ to be free from Int $C$. Thus, this unsolved question can be stated as follows.

4.1.2. Question. Is a crumpled cube $C$ a 3-cell if $\mathrm{Bd} C$ is free from Int $C$ ?

This question apparently first appeared in print in a paper by Hempel [72], and it is sometimes called the free surface problem. It is not known whether a surface would be free if it is locally free (see [32, p. 302]).

A sequence $\left\{S_{i}\right\}$ of 2-spheres is defined to converge homeomorphically to a 2 -sphere $S$ if there exists a sequence $\left\{h_{i}\right\}$ of homeomorphisms such that, for each $i, h_{i}(S)=S_{i}$ and $\left\{h_{i}\right\}$ converges to the identity homeomorphism on $S$. As indicated above in the brief outline of Cannon's proof of Theorem 4.1.1, the hypothesis of Theorem 4.1 .1 is equivalent to requiring that there be a sequence $\left\{S_{i}\right\}$ of disjoint 2-spheres in Int $C$ converging homeomorphically to Bd C. Thus we can state Theorem 4.1.1 in the following alternative form.

4.1.3. THEOREM. A crumpled cube $C$ is a 3-cell if there exists a sequence $\left\{S_{i}\right\}$ of 2-spheres in Int $C$ converging homeomorphically to $\mathrm{Bd} C$.

We can weaken the hypothesis of Theorem 4.1 .3 by requiring less than homeomorphic convergence of the sequence $\left\{S_{i}\right\}$. We say that the sequence $\left\{S_{i}\right\}$ is sequentially $1-U L C$ in Int $C$ if for any null sequence $\left\{J_{i}\right\}$ of simple closed curves, with $J_{i} \subset S_{i}$, there is a null sequence $\left\{U_{i}\right\}$ of subsets of Int $C$ such that $J_{i}$ is nullhomotopic in $U_{i}$. We notice that this is a weaker requirement than the notion of 1-regular convergence, defined by Whyburn [102], where it is further required that there be a null sequence $\left\{D_{i}\right\}$ of disks such that $D_{i} \subset S_{i}$ and $J_{i}=\mathrm{Bd} D_{i}$.

THEOREM 4.1.4. A crumpled cube $C$ is a 3-cell if there exists a sequence $\left\{S_{i}\right\}$ 
of 2-spheres in Int $C$ such that $\left\{S_{i}\right\}$ converges to $\mathrm{Bd} C,\left\{\operatorname{Int} S_{i}\right\}$ converges to Int $C$, and $\left\{S_{i}\right\}$ is sequentially $1-U L C$ in Int $C$.

The proof of this theorem, by Burgess and Loveland [33], is based mainly on Theorem 4.2.1.

4.2. LocAl SIMPLE CONNECTEDNESS. The next two theorems, which were first proved by Bing [16], have been highly important in the development of other criteria for tameness of 2-spheres. We readily see that Theorem 4.2.2 is a direct consequence of Theorem 4.2.1 [32, Theorem 6.4.2]. Bing's proof of Theorem 4.2.1 is based mainly upon Dehn's Lemma, Theorem 4.1.1, and the Side Approximation Theorem. His proof can be simplified, as outlined in [35], [32, pp. 303-304], by using Lister's form of the Side Approximation Theorem (Theorem 3.4). A set $V$ is defined to be locally simply connected (1-ULC) if for each $\varepsilon>0$ there exists a $\delta>0$ such that each loop in $V$ of diameter less than $\delta$ can be shrunk to a point in a subset of $V$ of diameter less than $\varepsilon$.

\subsubsection{Theorem. A crumpled cube $C$ is a 3-cell if Int $C$ is 1-ULC.}

We define Int $C$ to be locally simply connected at a point $p \in \operatorname{Bd} C$ if for each neighborhood $U$ of $p$ there is a neighborhood $V$ of $p$ such that $V \subset U$ and each loop in $V \cap I n t C$ can be shrunk to a point in $U \cap I n t C$.

4.2.2. Theorem. A crumpled cube $C$ is a 3-cell if Int $C$ is locally simply connected at each point of $\mathrm{Bd} C$.

If we replace "loop" with "unknotted polyhedral simple closed curve" in the above two definitions, we obtain definitions for Int $C$ to be "weakly 1-ULC" or "weakly locally simply connected" at points of $\mathrm{Bd} C$. An examination of Bing's proof in [16], and of the alternative proof in [35], [32, pp. 303-304], reveals that Theorems 4.2.1 and 4.2.2 hold true with this weaker version of local simple connectedness.

4.3. LOCAL SPANNING OF SPHERES. We say that the boundary of a crumpled cube $C$ can be locally spanned from Int $C$ if for each $\varepsilon>0$ and each $p \in \operatorname{Bd} C$ there exist disks $D$ and $D^{\prime}$ such that:

(1) $p \in$ Int $D \subset B d C$;

(2) Int $D^{\prime} \subset$ Int $C$;

(3) $\operatorname{Diam}\left(D \cup D^{\prime}\right)<\varepsilon$;

(4) $\mathrm{Bd} D=\mathrm{Bd} D^{\prime}$.

The following theorem is proved by using Theorem 4.2.1, the Tietze Extension Theorem, and some simple linking properties [28].

4.3.1. TheOREM. A crumpled cube $C$ is a 3-cell if $\mathrm{Bd} C$ can be locally spanned from Int $C$.

Cannon has proved a variation of this theorem where $\mathrm{Bd} C$ is locally singularly spanned from Int $C$. This can be stated as follows [42], [32, Theorem 6.5.9]: 
4.3.2. TheOREM. A crumpled cube $C$ in $E^{3}$ is a 3-cell if for each $p \in \mathrm{Bd} C$ and each $\varepsilon>0$ there is a map $f$ of a disk $D$ into an $\varepsilon$-subset of $C$ such that $f(D)-B d C=f($ Int $D)$ and $f(B d D)$ is tame and links $p$ in some $\varepsilon$ neighborhood of $\mathrm{p}$ on $\mathrm{Bd} C$.

Loveland [81, Theorem 3], [32, Theorem 6.5.8] has proved a variation of Theorem 4.3.1 where it is not required that $\mathrm{Bd} D=\mathrm{Bd} D^{\prime}$ but where $D^{\prime}$ is in Int $C$ and $B d D^{\prime}$ and $B d D$ are homotopically close in $E^{3}$. His proof also requires that $B d D$ be tame in $E^{3}$. It is not known whether it is necessary to impose tameness requirements in these two theorems of Cannon and Loveland. (See [32, p. 307], [42, p. 31], and [28, p. 96].)

4.4. Collars AND DEFormations OF SPHERES. If $C$ is a crumpled cube and $K$ is a subset of $\mathrm{Bd} C$, we say that $K$ can be collared in Int $C$ if there is a homeomorphism $h: K \times I \rightarrow C$ such that $h(x \times 0)=x$ for each $x \in K$ and $h(K \times t) \subset$ Int $C$ for each $t \in(0,1]$. We define Bd $C$ to be locally collared in Int $C$ if for each $p \in \mathrm{Bd} C$ there is an open subset $U$ of $\mathrm{Bd} C$ such that $p \in U$ and $U$ can be collared in Int $C$. We say that $K$ can be singularly collared in Int $C$, or that $K$ can be deformed into Int $C$, if there is a map $f: K \times I \rightarrow C$ such that $f(x \times 0)=x$ for each $x \in K$ and $f(K \times t) \subset$ Int $C$ for each $t \in(0,1]$.

The following two theorems are included in Brown's work on collaring of spheres [25], [26]. While his work included comparable theorems for $(n-1)$-spheres in $E^{n}$, the versions for $E^{3}$ had already been included in work by Bing [11] and Moise [89].

\subsubsection{TheOREM. A crumpled cube is a 3-cell if $\mathrm{Bd} \mathrm{C}$ can be collared in Int $C$. \\ 4.4.2. TheOREM. A crumpled cube $C$ is a 3-cell if $\mathrm{Bd} \mathrm{C}$ can be locally collared in Int $C$.}

Hempel [72] used the Sphere Theorem and Theorem 4.2.2 to generalize Theorem 4.4.1 as follows:

4.4.3. TheOREM. A crumpled cube $C$ is a 3-cell if $\mathrm{Bd} \mathrm{C}$ can be deformed into Int $C$.

Hempel also generalized Theorem 4.4 .2 by requiring only that $\mathrm{Bd} \mathrm{C}$ be locally deformed into $C$.

Other generalizations of Theorems 4.4.1, 4.4.2, and 4.4.3 require the collaring or deforming of some of the 1-dimensional subsets of $\mathrm{Bd} \mathrm{C}$. Eaton [62] proved the following theorem about collaring arcs on a 2-sphere.

4.4.4. TheOREM. A crumpled cube $C$ in $E^{3}$ is a 3-cell if each arc in $\mathrm{Bd} C$ is tame and has a collar in Int $C$.

It is necessary to require in Theorem 4.4.4 that every arc in $\mathrm{Bd} C$ be tame, for each arc in the sphere $S$ in Figure 4 can be collared in Int $S$.

4.4.5. THEOREM. A crumpled cube $C$ is a 3-cell if for each $p \in \mathrm{Bd} C$ there exists a nested sequence $\left\{D_{i}\right\}$ of disks in $\mathrm{Bd} C$ such that $p=\bigcap_{i=1}^{\infty} D_{i}=$ $\bigcap_{i=1}^{\infty}$ Int $D_{i}$ and $p \cup\left(\bigcup_{i=1}^{\infty} \mathrm{Bd} D_{i}\right)$ can be collared in Int $C$. 
This theorem can be proved by showing that Bd C can be locally spanned [28, Theorem 14]. It follows from Theorem 4.4.5 that $C$ is a 3-cell if each point in $\mathrm{Bd} C$ is an inaccessible point of a Sierpinski curve in $\mathrm{Bd} C$ which can be collared in Int $C$. (A Sierpiński curve is defined as follows: Let $D$ be a disk and $\left\{D_{i}\right\}$ a null sequence of disjoint disks in Int $D$ such that $\bigcup_{i=1}^{\infty} D_{i}$ is dense in $D$. The set $D-\bigcup_{i=1}^{\infty}$ Int $D_{i}$ is called a Sierpinski curve, and any point of this set not in $\mathrm{Bd} D \cup\left(\bigcup_{i=1}^{\infty} \mathrm{Bd} D_{i}\right)$ is called an inaccessible point [103], [18].) Cannon [42] has obtained the stronger theorem that $C$ is a 3-cell if each point in $\mathrm{Bd} \mathrm{C}$ is an inaccessible point of a Sierpiński curve in $\mathrm{Bd} C$ which can be singularly collared in Int $C$.

4.5. EXTENDING HOMEOMORPHISMS. First we consider a crumpled cube $C$ and a 3-cell $B^{3}$ such that for each $p \in \mathrm{BdC}$ and $q \in \mathrm{Bd} B^{3}$, there is a homeomorphism $h: p \cup \operatorname{Int} C \rightarrow q \cup \operatorname{Int} B^{3}$. Loveland [83] has observed that if $C$ is a crumpled cube which is different from a 3-cell and has a Fox-Artin type sphere as its boundary (Figure 4), then there is no point of $\mathrm{Bd} \mathrm{C}$ for which such a homeomorphism exists. On the other hand, he has proved that a 3-cell results where such a homeomorphism exists for each point of $\mathrm{Bd} \mathrm{C} \mathrm{[83].}$

4.5.1. THEOREM. If $C$ is a crumpled cube such that for each $p \in \mathrm{Bd} C$ there is a homeomorphism of $p \cup \operatorname{Int} C$ onto $q \cup \operatorname{Int} B^{3}$, where $B^{3}$ is a 3-cell and $q \in \mathrm{Bd} B^{3}$, then $C$ is a 3-cell.

Hempel [72] has proved the following theorem as a corollary to Theorem 4.4.3.

4.5.2. TheOREM. A crumpled cube $C$ is a 3-cell if there is a map $f: B^{3} \rightarrow C$ such that $f \mid \mathrm{Bd} B^{3}$ is a homeomorphism and $f\left(\right.$ Int $\left.B^{3}\right)=\operatorname{Int} C$.

There are some unsolved problems about extending homeomorphisms from Bd C onto itself to homeomorphisms of the crumpled cube $C$ onto itself.

4.5.3. Question. Is a crumpled cube $C$ a 3 -cell if for each $p, q \in \mathrm{Bd} C$ there is a homeomorphism of $C$ onto itself which carries $p$ to $q$ ?

4.5.4. Question. Is a crumpled cube $C$ a 3-cell if every homeomorphism of Bd $C$ onto itself can be extended to a homeomorphism of $C$ onto itself?

The first of these two questions was raised at the topology conference at the University of Georgia in 1961 [68], and two crumpled cubes described by Bing [15] and Gillman [69] were suggested as candidates for counterexamples. However, neither of these crumpled cubes is a counterexample for Question 4.5.4 [32, p. 312], and it has recently been shown that the one described by Gillman is not a counterexample to Question 4.5.3 [30]. It is not known whether the one described by Bing is a counterexample to Question 4.5.3. Bothe [23] and Shilepsky [96], [97] have described some wild simple closed curves in $E^{3}$ from which self-homeomorphisms can be extended to $E^{3}$. Shilepsky [96] has stated that a 2-sphere $S$ in $E^{3}$ is tame if it is homogeneous by isotopy, i.e., there is an isotopy $H$ of $E^{3}$ such that $H(S, t)=S$ for all $t$, $H(p, 0)=p$, and $H(p, 1)=q$. However, a proof of this has not yet appeared in print. 
4.6. Horizontal PLANAR SECTIONS. We define a horizontal section of a set $X$ in $E^{3}$ to be the intersection of $X$ with some horizontal plane. In 1922 , Alexander [1] suggested that a 2-sphere should be tame in $E^{3}$ if each of its horizontal sections is either a point or a simple closed curve. However, Bing [20] has shown, with an example, that Alexander's suggested proof is not valid without some additional hypothesis, such as requiring that $S$ be polyhedral [32, p. 324]. In 1969, Eaton [61] and Hosay [74] independently proved that all such spheres are tame in $E^{3}$. Their proofs involve showing that Int $C$ is $1-U L C$, so Dehn's Lemma and the Side Approximation Theorem are indirectly needed in their proofs.

4.6.1. Theorem. A crumpled cube $C$ in $E^{3}$ is a 3-cell if each horizontal section of $\mathrm{Bd} C$ is either a point or a simple closed curve. Furthermore, $\mathrm{Bd} C$ is tame in $E^{3}$.

By using Cannon's characterization of taming sets for 2-spheres (Theorem 7.1), Jensen [75] showed that the hypothesis of Theorem 4.6.1 can be weakened to require only that each horizontal section of $\mathrm{Bd} C$ be connected.

4.6.2. THEOREM. If $C$ is a crumpled cube in $E^{3}$ such that each horizontal section of $\mathrm{Bd} C$ is connected, then $C$ is a 3-cell. Furthermore, $\mathrm{Bd} C$ is tame in $E^{3}$.

Cannon [37], [38] used properties of $*$-taming sets, discussed in $\S 7$, to substitute some weaker requirements for the hypothesis that each horizontal section of $\mathrm{Bd} \mathrm{C}$ be connected. However, with one of these weaker hypotheses, it cannot be concluded that $\mathrm{Bd} C$ is tame in $E^{3}$. The crumpled cube described by Fox and Artin (Figure 4) illustrates the significance of the hypotheses of these theorems.

4.6.3. Theorem. A crumpled cube $C$ in $E^{3}$ is a 3-cell if no horizontal section of $\mathrm{Bd} C$ has a point as a component. Furthermore, $\mathrm{Bd} C$ is tame in $\mathrm{E}^{3}$.

4.6.4. TheOREM. A crumpled cube $C$ in $E^{3}$ is a 3-cell if each horizontal section of $\mathrm{Bd} C$ has at most four components. Furthermore, $\mathrm{Bd} C$ is tame in $\mathrm{E}^{3}$.

4.6.5. Theorem. A crumpled cube $C$ in $E^{3}$ is a 3-cell if each horizontal section of $\mathrm{Bd} C$ has at most five components.

4.7. VERTICAL LINEAR SECTIONS OF CRUMPLED CUBES. We see by an inspection of the 2-sphere indicated in Figure 3 that the intersection of Int $S$ with some vertical line is not connected and that some vertical line intersects $S$ in four points. Also, we see that the intersection of some vertical line with the 2-sphere $S^{\prime}$ indicated in Figure 4 consists of six points and that the intersection of Int $S^{\prime}$ with some vertical line has three components. Furthermore, we observe that there is no direction for "vertical lines" in which these numbers can be reduced. Figures 1 and 2 can similarly be used for these illustrations. These observations suggest the theorems discussed in this section. Jensen and Loveland [76] proved the first theorem of this type. 
4.7.1. TheOREM. A crumpled cube $C$ in $E^{3}$ is a 3-cell if the intersection of $\mathrm{Bd} C$ with each vertical line contains at most three points. Furthermore, $\mathrm{Bd} C$ is tame in $E^{3}$.

Cannon and Loveland [44] generalized Theorem 4.7.1 by restricting these vertical linear intersections to have no more than three components.

4.7.2. TheOREM. A crumpled cube $C$ in $E^{3}$ is a 3-cell if the intersection of $C$ with each vertical line has at most three components. Furthermore, $\mathrm{Bd} C$ is tame in $E^{3}$.

If we omit the conclusion that $\mathrm{Bd} C$ be tame we can permit the number of such components to be five [44], [84].

4.7.3. TheOREm. A crumpled cube $C$ in $E^{3}$ is a 3-cell if for each vertical line $L$, the set $L \cap \mathrm{Bd} C$ has at most five components. Furthermore, $\mathrm{Bd} C$ is locally tame from $E^{3}-C$ modulo a 0 -dimensional set.

Cannon and Loveland obtained the following three theorems about vertical linear intersections of the interior of a crumpled cube in $E^{3}$ [44].

4.7.4. Theorem. A crumpled cube $C$ in $E^{3}$ is a 3-cell if for each vertical line $L$, the set $L \cap I n t C$ is connected. Furthermore, $\mathrm{Bd} C$ is tame in $E^{3}$.

4.7.5. THEOREM. A crumpled cube $C$ in $E^{3}$ is a 3-cell if for each vertical line $L$, the set $L \cap I n t C$ has at most two components.

4.8. "Black Box" theOREMS FOR CRUMPLed CUBES. Some of the most interesting theorems about a crumpled cube $C$ in $E^{3}$ being a 3-cell are those in which the hypotheses include only "external" information about $C$. Thus, in some cases, we can "test" a crumpled cube $C$ from its exterior in $E^{3}$ and determine that $C$ is a 3-cell. These theorems have been proved by Cannon [37] using his characterization of $*$-taming sets for crumpled cubes in $E^{3}$. (See Theorem 7.5.)

4.8.1. THEOREM. A crumpled cube $C$ in $E^{3}$ is a 3-cell if each horizontal section of $E^{3}-C$ is connected.

4.8.2. Theorem. A crumpled cube $C$ in $E^{3}$ is a 3-cell if for each $p \in \mathrm{Bd} C$ there is a polyhedral 3-cell $K$ such that $K \cap C=p$.

The following theorem is a generalization of Theorem 4.8.2.

4.8.3. THEOREM. A crumpled cube $C$ in $E^{3}$ is a 3-cell if there exists a countable collection $\left\{L_{i}\right\}$ of straight lines in $E^{3}$ such that each $p \in \mathrm{Bd} C$ is in a closed straight line interval which is parallel to some $L_{i}$ and does not intersect Int $C$.

Examples of horned spheres described by Fort [66] and Bing [20] show the necessity of restricting the straight line intervals in the hypothesis of Theorem 4.8.3 to a countable number of directions.

4.9. MAPPING CRUMPLED CUBES ONTO 3-CElls. Armentrout [7], [8, Corollary 3] has proved two theorems about mappings which determine that a crumpled cube is a 3 -cell. 
4.9.1. Theorem. A crumpled cube $C$ is a 3-cell if there is a map $f$ of $C$ onto a 3-cell $B^{3}$ such that $f \mid B d C$ is a homeomorphism, $f(\operatorname{Int} C)=\operatorname{Int} B^{3}$, and only countably many points of $B^{3}$ have nondegenerate inverses under $f$.

The "countability" requirement can be removed provided it is required that inverses of points of Int $C$ be cellular; i.e., each inverse of a point under $f$ is the intersection of a nested sequence $\left\{K_{i}\right\}$ of 3-cells in Int $C$ such that $K_{i+1} \subset$ Int $K_{i}$.

4.9.2. TheOREM. A crumpled cube $C$ is a 3-cell if there is a cellular map $f$ of $C$ onto a 3-cell $B^{3}$ such that $f \mid B d C$ is a homeomorphism and $f(\operatorname{Int} S)=\operatorname{Int} B^{3}$.

Boyd [24] has shown that "cellular" cannot be replaced with "monotone" in the hypothesis of Theorem 4.9.2.

5. Tame spheres in $E^{3}$. As indicated with their statements, the hypotheses of Theorems 4.6.1-4.6.4, 4.7.1, 4.7.2, and 4.7.4 imply that Bd C is tame in $E^{3}$. Thus, without any additional hypotheses, these theorems give conditions under which a 2-sphere is tame in $E^{3}$. Although we do not include revisions of their statements, all of the other theorems in $\$ 4$ except Theorems 4.6.5 and 4.7.3 can be adapted to theorems about tame 2-spheres in $E^{3}$ by requiring that $C$ be in $E^{3}$ and that the hypotheses be satisfied for $E^{3}$-Int $C$ as well as for $C$. The wild 2-sphere indicated in Figure 3 satisfies the hypotheses of Theorems 4.6.5 and 4.7.3.

The following theorem is a corollary of Theorem 4.3.1. (Harrold [71] had previously proved such a theorem with an additional hypothesis which is equivalent to requiring that $S^{\prime} \cap S$ be a tame simple closed curve.)

5.1. THEOREM. A 2-sphere $S$ is tame in $E^{3}$ if for each $p \in S$ and each $\varepsilon>0$ there is a 2-sphere $S^{\prime}$ such that $p \in \operatorname{Int} S^{\prime}$, Diam $S^{\prime}<\varepsilon$, and $S^{\prime} \cap S$ is a simple closed curve.

Some more general theorems of this type are discussed in [32, pp. 306-307]. More recently, Cannon [40, Corollary 5.3] has proved the following general theorem of this type.

5.2. THEOREM. A 2-sphere $S$ is tame in $E^{3}$ if for each $p \in S$ and each $\varepsilon>0$ there is a 2-sphere $S^{\prime}$ such that $p \in \operatorname{Int} S^{\prime}$, Diam $S^{\prime}<\varepsilon$, and $S^{\prime} \cap S$ is connected.

6. Tame subsets of 2 -spheres in $E^{3}$. We define a closed subset $X$ of a 2-sphere in $E^{3}$ to be tame if $X$ is a subset of some tame 2-sphere. Loveland [81] proved one of the first important theorems about tame subsets of 2-spheres in $E^{3}$ by showing that a closed subset $X$ of a 2 -sphere $S$ in $E^{3}$ is tame if $S$ can be side approximated, as in Theorem 3.4, missing $X$. We say that a 2-sphere $S$ in $E^{3}$ can be side approximated missing the closed subset $X$ of $S$ if for each $\varepsilon>0$ there exist $\varepsilon$-homeomorphisms $g$ and $h$ satisfying the requirements of the conclusion of Theorem 3.4 such that $X \subset S-\bigcup_{i=1}^{n} D_{i}$, where the $D_{i}$ 's are the disks in $S$ identified in that theorem. 
6.1. TheOREM. A closed subset $X$ of a 2-sphere $S$ in $E^{3}$ is tame if $S$ can be side approximated missing $X$. Furthermore, for each $\varepsilon>0$, there is a tame continuum $K$ in $S$ such that $X \subset K$ and $K=S-\bigcup_{i=1}^{\infty}$ Int $D_{i}$, where $\left\{D_{i}\right\}$ is a null sequence of disjoint disks in $S-X$.

The converse of the first part of this theorem is not true, as the set consisting of the point $p$ of the 2-sphere $S$ described in Figure 4 is tame, but $S$ cannot be side approximated from Int $S$ missing $p$. However, Cannon [39] has shown that if we do not require that $X$ be between two side approximations of $S$, then we can obtain a converse theorem. We say that a closed subset $X$ of a 2 -sphere $S$ in $E^{3}$ can be wedged between homeomorphic approximations of $S$ if for each $\varepsilon>0$ there exist homeomorphisms $h_{1}$ and $h_{2}$ of $S$ such that $X \subset$ Int $h_{1}(S) \cap$ Ext $h_{2}(S)$ and, for $i=1,2, h_{i}$ moves no point more than a distance $\varepsilon$.

6.2. Theorem. A closed subset $X$ of a 2-sphere $S$ in $E^{3}$ is tame if and only if $X$ can be wedged between homeomorphic approximations of $S$.

There is a converse to Theorem 6.1 provided it is required that $X$ have no degenerate component [34, Theorem 1.1 and Corollary 5.4]. This characterization of tame subsets requires Cannon's characterization of taming sets discussed in $\$ 7$.

6.3. THEOREM. If $X$ is a closed subset of a 2-sphere $S$ in $E^{3}$ and each component of $X$ is nondegenerate, then $S$ is tame if and only if $S$ can be side approximated missing $X$.

By considering the special case where $X=S$, we see that each of the above three theorems is an adaptation of Theorem 4.1.1 for closed subsets of $S$. Cannon [39] has shown that Theorem 4.2.1 also can be adapted to closed subsets of $S$. Let $X$ be a closed subset of a 2 -sphere $S$ in $E^{3}$. We say that $E^{3}-S$ is $1-U L C$ in $E^{3}-X$ if for each $\varepsilon>0$ there is a $\delta>0$ such that each $\delta$-loop in $E^{3}-S$ can be shrunk to a point in an $\varepsilon$-subset of $E^{3}-X$.

6.4. THEOREM. A closed subset $X$ of a 2-sphere $S$ in $E^{3}$ is tame if and only if $E^{3}-S$ is $1-U L C$ in $E^{3}-X$.

The following theorems can be proved as consequences of the above theorems [82].

6.5. Theorem. A closed subset $X$ of a 2-sphere $S$ in $E^{3}$ is tame if $E^{3}-S$ is locally simply connected at each point of $X$.

6.6. THEOREM. A closed subset $X$ of a 2-sphere $S$ in $E^{3}$ is tame if $S$ can be locally spanned at each point of $X$ from each component of $E^{3}-S$.

We say that a continuum $X$ in $E^{3}$ can be described with trees of 3-cells if for each $\varepsilon>0$, there exists a 3 -cell $K$ such that $X \subset$ Int $K$ and $K$ is the union of the elements of a tree of 3-cells each of diameter less than $\varepsilon$.

6.7. THEOREM. If $X$ is a subcontinuum of a 2-sphere $S$ in $E^{3}$ and $X$ can be described with trees of 3-cells, then $X$ is tame [31]. 
A version of this theorem where $X$ is not required to be connected is included in [31]. We observe that Theorem 4.3.2, about singular spanning of 2-spheres, can be adapted to closed subsets of 2-spheres.

7. Taming sets for spheres and crumpled cubes. In this section we discuss two concepts, taming sets and *-taming sets, which are used in the proofs of some of the theorems in $\S 4$. We define a compact set $X$ to be a taming set for 2-spheres in $E^{3}$ if (1) $X$ is a subset of some 2-sphere in $E^{3}$ and (2) a 2-sphere $S$ is tame provided it contains $X$ and $S-X$ is locally tame. Several theorems which identified certain types of sets as taming sets during the period 1960-1969 are summarized in [32, pp. 319-321]. One of the first such theorems, proved by Doyle and Hocking [59], stated that every tame arc in $E^{3}$ is a taming set for 2 -spheres; i.e., if a 2 -sphere $S$ in $E^{3}$ is locally tame modulo a tame arc in $S$, then $S$ is tame. In 1969, Cannon [34] gave a complete characterization of taming sets for 2-spheres in $E^{3}$.

7.1. Theorem. A closed set $X$ in a 2 -sphere in $E^{3}$ is a taming set for 2 -spheres if and only if $X$ is tame and has no degenerate component.

7.2. COROllary. Every nondegenerate tame subcontinuum of a 2-sphere in $E^{3}$ is a taming set for 2-spheres.

7.3. TheOREM. A closed subset $X$ of a 2-sphere in $E^{3}$ is a taming set if $X$ is the union of a countable number of taming sets.

If we consider a crumpled cube $C$ in a 3 -sphere $S^{3}$, then $S^{3}-$ Int $C$ is a crumpled cube which we denote by $C^{*}$. A *-taming set is defined to be a compact set $X$ with the following property: If $C$ is a crumpled cube in $S^{3}$, $X \subset C$, and $\mathrm{Bd} C$ is locally tame at each point of $\mathrm{Bd} C-X$, then $C^{*}$ is a 3-cell. In this definition, it is not required that $X$ be a subset of a 2 -sphere in $E^{3}$.We notice that the above definition can be adapted for $E^{3}$. Where $X$ is a subset of a 2-sphere, the two taming concepts defined above have been shown by Cannon [36, Corollary 3.8] to be related as follows:

7.4. THEOREM. A closed subset $X$ of a 2 -sphere $S$ in $E^{3}$ is a $*$-taming set if and only if it is a taming set.

The following characterization [36, Corollary 3.1] does not require that $X$ be a subset of a 2-sphere.

7.5. THEOREM. A closed subset $X$ of $E^{3}$ is a*-taming set if and only if for each crumpled cube $C$ which contains $X, C^{*}-X$ is 1-ULC.

The following theorem [29, Theorem 6], [36, Theorem 4.1] identifies a *-taming set which is not a subset of a 2-sphere.

7.6. TheOREM. A crumpled cube $X$ in $E^{3}$ is a $*$-taming set if $X^{*}$ is a 3-cell.

As with taming sets (Theorem 7.3), *-taming sets are preserved under closed countable unions [36, Theorem 3.7].

7.7. THEOREM. A closed subset of $E^{3}$ is a -taming set if it is the union of a countable number of *-taming sets. 
8. Extensions to 2-manifolds in a 3-manifold. Many of the theorems discussed in $\S \S 4,5$ and 6 about tameness of 2-spheres, or subsets of them, can be adapted to 2-manifolds in a 3-manifold. While there exists a 2-manifold $M^{2}$ in a 3-manifold $M^{3}$ such that some disk in $M^{2}$ is not a subset of a 2-sphere in $M^{3}$ [16], it has been shown by Bing [16, Theorem 5] that each $p \in M^{2}$ is in a Euclidean neighborhood $U$ in $M^{3}$ such that some disk in $M^{2}$ contains $p$ in its interior and is a subset of a 2 -sphere in $U$. This enables us to extend all local properties discussed in $\S \S 4,5$ and 6 to 2 -manifolds in $M^{3}$. Cannon and Loveland [44] extended some of the theorems about vertical linear sections of 2-spheres to 2-manifolds in $E^{3}$. Cannon's characterization of taming sets for 2-spheres in $E^{3}$ extends to 2-manifolds in a 3-manifold [32, Theorems 11.2.1, 11.2.2]. A further discussion of tame 2-manifolds in a 3-manifold can be found in [32, pp. 333-337].

9. Other examples of wild spheres. The methods used in the construction of the three types of wild spheres discussed in $\$ 1$ can be varied to obtain other wild spheres. Some different types of horned spheres have been described by Ball [9] and L. O. Cannon [45], and uncountably many different embeddings of 2-spheres which are locally tame except at one point have been described by Kinoshita and Sikkema [77] and McPherson [87]. Martin [86] used the existence of infinitely many different such embeddings to describe a rigid 2-sphere in $E^{3}$, i.e., a sphere $S$ such that no homeomorphism of $S$ onto itself, other than the identity homeomorphism, can be extended to $E^{3}$. The following question about rigid spheres remains unanswered.

9.1. Question. Does every rigid 2 -sphere in $E^{3}$ contain a wild arc?

Bing [15] described a wild 2-sphere in which every arc is tame, and Gillman [69] modified Bing's example so that each complementary domain of the sphere, in $S^{3}$, is an open 3-cell. Alford [4] described other examples with variations in Bing's construction..

Other examples of wild 2-spheres in $E^{3}$ have been obtained by "sewing" two crumpled cubes together on their boundaries. Bing [10] used this method to obtain a 2 -sphere $S$ in $S^{3}$ such that each crumpled cube having $S$ as its boundary is homeomorphic with the crumpled cube in Figure 2 and $S$ is locally wild from both sides on the same Cantor set. The following theorem, which is a direct consequence of a more general theorem by Daverman and Eaton [58], suggests the extensive variations in types of wild embeddings of 2 -spheres in $S^{3}$.

9.2. THEOREM. If $C_{1}$ and $C_{2}$ are two crumpled cubes, then there is a 2-sphere $S$ in $S^{3}$ such that one of the crumpled cubes bounded by $S$ is $C_{1}$ and the other is $\mathrm{C}_{2}$.

Further discussions of sewings of crumpled cubes can be found in [32, pp. 327-333] and other papers cited therein.

10. Higher dimensional spaces. We briefly mention work in higher dimensional Euclidean spaces on problems similar to those discussed for $E^{3}$ in the preceding sections. The definition of tame 2-spheres in $E^{3}$ is extended to 
$(n-1)$-spheres in $E^{n}$. Although the term "flat" has frequently been used instead of "tame" for higher dimensional spaces, we shall continue, in this section, to use "tame." Daverman [56] has recently presented a more complete summary of conditions which imply that an $(n-1)$-sphere is tame in $E^{n}$.

First, Brown's work on collaring and local collaring of spheres is valid in all dimensions, i.e., for each $n, S \cup \operatorname{Int} S$ is an $n$-cell if the $(n-1)$-sphere $S$ can be locally collared in Int $S$ [26].

Cernavskii [47] and Daverman [50] independently have extended, to all dimensions greater than four, Bing's theorem that a 2 -sphere in $E^{3}$ is tame if its complement is $1-U L C$.

10.1. Theorem. For $n \neq 4$, an $(n-1)$-sphere $S$ is tame in $E^{n}$ if $E^{n}-S$ is 1-ULC.

Thus, as in three dimensions, the following theorem is valid.

10.2. TheOREM. For $n \neq 4$, an $(n-1)$-sphere $S$ is tame in $E^{n}$ if $E^{n}-S$ is locally simply connected at each point of $S$.

The proof given for [28, Theorem 7], to show that local spanning implies local simple connectedness, is valid in all dimensions. Thus Theorem 10.2 implies

10.3. Theorem. For $n \neq 4$, an $(n-1)$-sphere $S$ is tame in $E^{n}$ if $S$ can be locally spanned.

We readily see that Theorem 10.1 implies

10.4. Theorem. For $n \neq 4$, an $(n-1)$-sphere $S$ is tame in $E^{n}$ if $S$ can be homeomorphically approximated in each component of $E^{n}-S$.

As in dimension three [32, Theorem 3.6.1], this theorem then has the following corollary.

10.5. THEOREM. For $n \neq 4$, there do not exist uncountably many disjoint wild $(n-1)$-spheres in $E^{n}$.

Daverman [54] has also proved the following theorem as a consequence of Theorem 10.1. This theorem has been proved independently by Bryant and Lacher [27] and extended to other codimensions.

10.6. THEOREM. If $n \neq 4$, an $(n-1)$-sphere $S$ is tame in $E^{n}$ if $S$ can be locally deformed into each component of $E^{n}-S$.

It is interesting to notice that for $n \geqq 5$, Theorem 10.1 was proved independently of Theorem 10.4 and that Theorem 10.4 then followed as a corollary to Theorem 10.1. On the other hand, for $n=3$, Theorem 10.4 was proved first and Theorem 10.1 then followed from Dehn's Lemma, the Side Approximation Theorem, and Theorem 10.4. Also, it should be emphasized that, unlike the situation in dimension three, the proofs now available for Theorems 10.1-10.4 and 10.6 require that the hypotheses be satisfied in both 
components of $E^{n}-S$, where $n \geqq 5$. Thus the following question remains unanswered.

10.7. Question. If $S$ is an $(n-1)$-sphere in $E^{n}$, is the crumpled $n$-cube $S \cup I n t S$ an $n$-cell if Int $S$ is $1-U L C$ ?

Daverman [49] has adapted Theorem 4.6.1 to higher dimensions as follows:

10.8. THEOREM. For $n \neq 4$, an $(n-1)$-sphere $S$ is tame in $E^{n}$ if each "horizontal" (n-1)-plane $P$ intersects $S$ either in a point or an $(n-2)$-sphere which is tame in both $P$ and in $S$.

In a similar manner, Daverman [55] has adapted Theorem 4.4.4, about collaring arcs in a sphere, to conditions which imply that $(n-1)$-spheres in $E^{n}$ $(n>4)$ are tame. Cannon [40, Corollary 5.3] proved Theorem 5.2 by showing that $E^{3}-S$ is $1-U L C$. He also proved, with the same hypothesis adapted for an $(n-1)$-sphere $S$ in $E^{n}$, that $E^{n}-S$ is $1-U L C$ provided it is further required that each component of $S-S^{\prime}$ be simply connected. Thus, with Theorem 10.1 , we see that Theorem 5.2 can be extended to $E^{n}$ as follows:

10.9. THEOREM. For $n \neq 4$, an $(n-1)$-sphere $S$ is tame in $E^{n}$ if for each $p \in S$ and each $\varepsilon>0$ there is an $(n-1)$-sphere $S^{\prime}$ such that $p \in \operatorname{Int} S^{\prime}$, Diam $S^{\prime}<\varepsilon$, $S^{\prime} \cap S$ is connected, and each component of $S-S^{\prime}$ is simply connected.

In [56], Daverman cites some references and discusses the construction of wild $(n-1)$-spheres in $E^{n}$ by various methods including suspensions of wild $(n-2)$-spheres, construction of wild Cantor sets, Cartesian products of lines and certain decompositions of $E^{n-1}$, and higher dimensional versions of Alexander's horned sphere. By using the suspension method [56, p. 157] with the Fox-Artin sphere (Figure 3), we can obtain a wild (n-1)-sphere $S$ in $E^{n}$ such that $S$ is locally tame modulo an $(n-3)$-cell which is tame in both $S$ and $E^{n}$. It is interesting, however, that there is no higher dimensional version of the Fox-Artin sphere such that the sphere is locally tame modulo a 0dimensional set. Cantrell [46] showed that, for $n>3$, there is no wild $(n-1)$-sphere in $E^{n}$ which is locally tame modulo one point. Kirby [78] proved the following more general theorem.

10.10. THEOREM. If $n \geqq 4$, an $(n-1)$-sphere $S$ is tame in $E^{n}$ if $S$ is locally tame modulo a compact 0 -dimensional set which is tame in both $E^{n}$ and $S$.

Examples of higher dimensional horned spheres described by Daverman [53] are wild at points of Cantor sets which are tame in $E^{n}$ but not in $S$, and examples mentioned by Kirby [78] are wild at points of Cantor sets which are tame in $S$ but not in $E^{n}$.

We could rephrase Theorem 10.10 so that it states that a compact 0 -dimensional set $X$ is a taming set for the $(n-1)$-sphere $S$ in $E^{n}, n>3$, provided $X$ is tame in both $E^{n}$ and $S$. Beyond this, very little is known about taming sets for $(n-1)$-spheres in $E^{n}$. Also, for $n>3$, very little progress has been made in determining conditions which imply that a closed subset of an $(n-1)$-sphere in $E^{n}$ is tame. Daverman [53] has described examples where Theorem 7.6, about crumpled cubes which are $*$-taming sets, fails in higher 
dimensions. This suggests that there are difficulties in developing a theory of *-taming sets for higher dimensions. Also, Theorem 4.7.1 fails in higher dimensions as Daverman [57] has described a wild (n-1)-sphere $S$ in $E^{n}$ such that no "vertical" line intersects $S$ in more than two points. These examples suggest that there are probably other peculiarities in higher dimensional spaces relative to what occurs in $E^{3}$. Daverman [56] discusses briefly the current lack of suitable results about polyhedral approximations of $(n-1)$-spheres in $E^{n}$, and he has shown [52], using one of his examples in [51], that the Side Approximation Theorem cannot be extended to higher dimensions with a retention of the requirement that the intersection of $S$ with the approximating sphere be in the interiors of disjoint small $(n-1)$-cells in $S$. However, he has conjectured that it might be possible to replace these small open $(n-1)$-cells with disjoint small open sets in $S[56,5.5]$. Seebeck [93] and Sher [95] have extended Bing's theorem [17, Theorem 9], about approximating arcs in 2 -cells in $E^{3}$ with tame arcs, to arcs in 2-cells in $E^{n}, n \geqq 4$. Daverman [51, Theorem 5.2] has shown that there is not a similar result about approximating disks in $k$-cells in $E^{n}, 3 \leqq k \leqq n$, with tame disks. Also, he has obtained some preliminary results about sewings of $n$-cubes [57], and he discusses, in [56], the lack of suitable results about sewings in higher dimensions. In particular, Theorem 9.2 has not yet been extended to higher dimensions. He lists many questions [56] about $E^{n}$ which have been answered for $E^{3}$ but not for $E^{n}$, $n>3$. Finally, we wish to emphasize that $E^{4}$ remains an outcast among Euclidean spaces as very few of the problems discussed in this paper have been solved for $E^{4}$.

\section{REFERENCES}

1. J. W. Alexander, On the subdivision of 3-space by a polyhedron, Proc. Nat. Acad. Sci. U.S.A. 10 (1924), 6-8.

2. - An example of a simply connected surface bounding a region which is not simply connected, Proc. Nat. Acad. Sci. U.S.A. 10 (1924), 8-10.

3. - Remarks on a point set constructed by Antoine, Proc. Nat. Acad. Sci. U.S.A. 10 (1924), 10-12.

4. W. R. Alford, Some "nice" wild 2-spheres in $E^{3}$, Topology of 3-Manifolds and Related Topics (Proc. Univ. of Georgia Inst., 1961), Prentice-Hall, Englewood Cliffs, N.J., 1962, pp. 29-33. MR 25 \#4504.

5. M. L. Antoine, Sur la possibilité d'étendre l'homéomorphie de deux figures a leurs voisinages, C. R. Acad. Sci. Paris 171 (1920), 661-663.

6. _- Sur l'homéomorphie de deux figures et de leurs voisinages, J. Math. Pures Appl. 86 (1921), 221-325.

7. Steve Armentrout, Upper semi-continuous decompositions of $E^{3}$ with at most countably many non-degenerate elements, Ann. of Math. (2) 78 (1963), 605-618. MR 27 \#6255.

8. - Concerning cellular decompositions of 3-manifolds with boundary, Trans. Amer. Math. Soc. 137 (1969), 231-236. MR 38 \#5224.

9. B. J. Ball, The sum of two solid horned spheres, Ann. of Math. (2) 69 (1959), 253-257. MR 21 \#321.

10. R. H. Bing, A homeomorphism between the 3-sphere and the sum of two solid horned spheres, Ann. of Math. (2) 56 (1952), 354-362. MR 14, 192.

11. - Locally tame sets are tame, Ann. of Math. (2) 59 (1954), 145-158. MR 15, 816.

12. - Approximating surfaces with polyhedral ones, Ann. of Math. (2) 65 (1957), 456-483. MR 19, 300. 
13. R. H. Bing, Conditions under which a surface in $E^{3}$ is tame, Fund. Math. 47 (1959), 105-139. MR 21 \#5954.

14. - An alternative proof that 3-manifolds can be triangulated, Ann. of Math. (2) 69 (1959), 37-65. MR 20 \#7269.

15. — A A wild surface each of whose arcs is tame, Duke Math. J. 28 (1961), 1-15. MR 23 \#A630.

16. - A surface is tame if its complement is 1-ULC, Trans. Amer. Math. Soc. 101 (1961), 294-305. MR 24 \#A1117.

17. — Each disk in $E^{3}$ contains a tame arc, Amer. J. Math. 84 (1962), 583-590. MR 26 \#4331.

18. —- Each disk in $E^{3}$ is pierced by a tame arc, Amer. J. Math. 84 (1962), 591-599. MR $26 \# 4332$.

19. - Approximating surfaces from the side, Ann. of Math. (2) 77 (1963), 145-192. MR $27 \# 731$.

20. - Spheres in $E^{3}$, Amer. Math. Monthly 71 (1964), 353-364. MR 29 \#2789.

21. _ Improving the side approximation theorem, Trans. Amer. Math. Soc. 116 (1965), 511-525. MR 33 \#704.

22. - Improving the intersections of lines and surfaces, Michigan Math. J. 14 (1967), 155-159. MR 34 \#6743.

23. H. G. Bothe, Ein homogen wilder Knoten, Fund. Math. 60 (1967), 271-283. MR 35 \#7327.

24. William S. Boyd, Jr., Repairing embeddings of 3-cells with monotone maps of $E^{3}$, Trans. Amer. Math. Soc. 161 (1971), 123-144. MR 43 \#8064.

25. Morton Brown, A proof of the generalized Schoenflies theorem, Bull. Amer. Math. Soc. 66 (1960), 74-76. MR 22 \#8470b.

26. — Locally flat imbeddings of topological manifolds, Ann. of Math. (2) 75 (1962), 331-341. MR 24 \#A3637.

27. J. L. Bryant and R. C. Lacher, Embeddings with mapping cylinder neighborhoods, Topology (to appear).

28. C. E. Burgess, Characterizations of tame surfaces in $E^{3}$, Trans. Amer. Math. Soc. 114 (1965), 80-97. MR 31 \#728.

29. - Pairs of 3-cells with intersecting boundaries in $E^{3}$, Amer. J. Math. 88 (1966), 309-313. MR 33 \#4909.

30. — Extending self homeomorphisms of a wild sphere described by Gillman, Duke Math. J. 39 (1972), 725-728. MR 47 \#2588.

31. C. E. Burgess and J. W. Cannon, Tame subsets of spheres in $E^{3}$, Proc. Amer. Math. Soc. 22 (1969), 395-401. MR 39 \#3469.

32. —_Embeddings of surfaces in $E^{3}$, Rocky Mountain J. Math. 1 (1971), No. 2, 259-344. MR 43 \#4008.

33. C. E. Burgess and L. D. Loveland, Sequentially 1-ULC surfaces in $E^{3}$, Proc. Amer. Math. Soc. 19 (1968), 653-659. MR 37 \#3546.

34. J. W. Cannon, Characterization of taming sets on 2-spheres, Trans. Amer. Math. Soc. 147 (1970), 289-299. MR 41 \#2644.

35. - Sets which can be missed by side approximations to spheres, Pacific J. Math. 34 (1970), 321-334. MR 42 \#2447.

36. — - *-Taming sets for crumpled cubes. I: Basic properties, Trans. Amer. Math. Soc. 161 (1971), 429-440. MR 43 \#8065.

37. - *-Taming sets for crumpled cubes. II: Horizontal sections in closed sets, Trans. Amer. Math. Soc. 161 (1971), 441-446. MR 43 \#8066.

38. — - *-Taming sets for crumpled cubes. III: Horizontal sections in 2-spheres, Trans. Amer. Math. Soc. 161 (1971), 447-456. MR 43 \#8067.

39. - Characterizations of tame subsets of 2-spheres in $E^{3}$, Amer. J. Math. 94 (1972), 173-188. MR 45 \#5980.

40. - ULC properties in neighborhoods of embedded surfaces and curves in $E^{3}$, Canad. J. Math. 25 (1973), 31-73. MR 47 \#2589. 
41. J. W. Cannon, New proofs of Bing's approximation theorems for surfaces, Pacific J. Math. 46 (1973), 361-379. MR 48 \#7289.

42. —_ Singular side approximations for 2-spheres in $E^{3}$, Illinois J. Math. 18 (1974), $27-36$.

43. _- Piercing disks with tame arcs, Michigan Math. J. 20 (1974), 345-347.

44. J. W. Cannon and L. D. Loveland, A 2-sphere in $E^{3}$ with vertically connected interior is tame, Trans. Amer. Math. Soc. 195 (1974), 345-355.

45. L. O. Cannon, Sums of solid horned spheres, Trans. Amer. Math. Soc. 122 (1966), 203-228. MR 32 \#8322.

46. J. C. Cantrell, Almost locally flat embeddings of $S^{n-1}$ in $S^{n}$, Bull. Amer. Math. Soc. 69 (1963), 716-718. MR 27 \#4237.

47. A. V. Černavskiǐ, Coincidence of local flatness and local simple connectedness for embeddings of (n-1)-dimensional manifolds in $n$-dimensional manifolds, Mat. Sb. 91 (133) (1973), 279-286=Math. USSR Sb. 20 (1973), 297-304.

48. R. J. Daverman, A new proof for the Hosay-Lininger theorem about crumpled cubes, Proc. Amer. Math. Soc. 23 (1969), 52-54. MR 39 \#7578.

49. - Slicing theorems for $n$-spheres in Euclidean $(n+1)$-space, Trans. Amer. Math. Soc. 166 (1972), 479-489. MR 45 \#4422.

50. - Locally nice codimension one manifolds are locally flat, Bull. Amer. Math. Soc., 79 (1973), 410-413. MR 47 \#9628.

51. - On the scarcity of tame disks in certain wild cells, Fund. Math. 79 (1973), 63-77. MR 48 \#5085.

52. - Approximating polyhedra in codimension one spheres embedded in $S^{n}$ by tame polyhedra, Pacific J. Math. 51 (1974), 417-426.

53. - Wild spheres in $E^{n}$ that are locally flat modulo tame Cantor sets, Trans. Amer. Math. Soc. 206 (1975), 347-359.

54. - Singular regular neighborhoods and local flatness in codimension one, Proc. Amer.

Math. Soc. (to appear).

55. —- Flattening a codimension one manifold by piercing with cells (to appear).

56. - A summary of results and problems concerning flatness of codimension one spheres in $E^{n}$, Geometrical Topology, Proc. Geometrical Topology Conf. (Park City, Utah, 1974), pp. $156-165$.

57. - Sewings of closed $n$-cell complements (to appear).

58. R. J. Daverman and W. T. Eaton, A dense set of sewings of two crumpled cubes yields $S^{3}$, Fund. Math. 65 (1969), 51-60. MR 39 \#4821.

59. P. H. Doyle and J. G. Hocking, Some results on tame discs and spheres in $E^{3}$, Proc. Amer.

Math. Soc. 11 (1960), 832-836. MR 23 \#A4133.

60. W. T. Eaton, Side approximations in crumpled cubes, Duke Math. J. 35 (1968), 707-719. MR 38 \#694.

61. Cross sectionally simple spheres, Bull. Amer. Math. Soc. 75 (1969), 375-378. MR 39 \#957.

62. _ Taming a surface by piercing with disks, Proc. Amer. Math. Soc. 22 (1969), 724-727. MR 39 \#7579.

63. - The sum of solid spheres, Michigan Math. J. 19 (1972), 193-207. MR 46 \#8227.

64. —, A two sided approximation theorem for 2-spheres, Pacific J. Math. 44 (1973), 461-487. MR 47 \#9606.

65. — Applications of a mismatch theorem to decomposition spaces, Fund. Math. (to appear).

66. M. K. Fort, Jr., A wild sphere which can be pierced at each point by a straight line segment, Proc. Amer. Math. Soc. 14 (1963), 994-995. MR 28 \#2528.

67. R. H. Fox and E. Artin, Some wild cells and spheres in three-dimensional space, Ann. of Math. (2) 49 (1948), 979-990. MR 10, 317.

68. D. S. Gillman, Tame subsets of 2-spheres in $E^{3}$, Topology of 3-manifolds and Related Topics (Proc. Univ. of Georgia Inst., 1961), Prentice-Hall, Englewood Cliffs, N.J., 1962, pp. 26-28. MR 25 \#4503. 
69. D. S. Gillman, Note concerning a wild sphere of Bing, Duke Math. J. 31 (1964), 247-254. MR $28 \# 4525$.

70. O. G. Harrold, Jr., Locally tame curves and surfaces in three-dimensional manifolds, Bull. Amer. Math. Soc 63 (1957), 293-305. MR 19, 568.

71. - Locally peripherally unknotted surfaces in $E^{3}$, Ann. of Math. (2) 69 (1959), 276-290. MR 21 \#4399a.

72. J. P. Hempel, A surface in $S^{3}$ is tame if it can be deformed into each complementary domain, Trans. Amer. Math. Soc. 111 (1964), 273-287. MR 28 \#3409.

73. David W. Henderson, Extension of Dehn's Lemma and the loop theorem, Trans. Amer. Math. Soc. 120 (1965), 448-469. MR 32 \#4686.

74. Norman Hosay, A proof of the slicing theorem for 2-spheres, Bull. Amer. Math. Soc. 75 (1969), 370-374. MR 39 \#956.

75. R. A. Jensen, Cross sectionally connected 2-spheres are tame, Bull. Amer. Math. Soc. 76 (1970), 1036-1038. MR 42 \#5241.

76. R. A. Jensen and L. D. Loveland, Surfaces of vertical order 3 are tame, Bull. Amer. Math. Soc. 76 (1970), 151-154. MR 40 \#3520.

77. S. Kinoshita and C. D. Sikkema, Penetration indices of arc and ball pairs and uncountably many quasi-translations of the 3-sphere, Michigan Math. J. 20 (1974), 309-319.

78. R. C. Kirby, On the set of non-locally flat points for a submanifold of codimension one, Ann. of Math. (2) 88 (1968), 281-290. MR 38 \#5193.

79. F. M. Lister, Simplifying intersections of disks in Bing's side approximation theorem, Pacific J. Math. 22 (1967), 281-295. MR 35 \#7317.

80. - Tame boundary sets of crumpled cubes in $E^{3}$, Proc. Amer. Math. Soc. 25 (1970), 377-378. 41 \#2647.

81. L. D. Loveland, Tame subsets of spheres in $E^{3}$, Pacific J. Math. 19 (1966), 489-517. MR 37 \#903.

82. - Tame surfaces and tame subsets of spheres in $E^{3}$, Trans. Amer. Math. Soc. 123 (1966), 355-368. MR 33 \#7990.

83. - Tameness implied by extending a homeomorphism to a point, Proc. Amer. Math. Soc. 23 (1969), 287-293. MR 40 \#3521.

84. — Vertically countable spheres and their wild sets, Pacific J. Math. 50 (1974), 521-529.

85. J. M. Martin, A note on uncountably many disks, Pacific J. Math. 13 (1964), 1331-1333. MR 27 \#6252.

86. — A rigid sphere, Fund. Math. 59 (1966), 117-121. MR 36 \#7122.

87. J. M. McPherson, Wild arcs in three-space. I: Families of Fox-Artin arcs, Pacific J. Math. 45 (1973), 585-598.

88. E. E. Moise, Affine structures in 3-manifolds. V: The triangulation theorem and Hauptvermutung, Ann. of Math. (2) 56 (1952), 96-114. MR 14, 72.

89. _ Affine structures in 3-manifolds. VIII: Invariance of the knot-types; local tame imbedding, Ann. of Math. (2) 59 (1954), 159-170. MR 15, 889.

90. C. D. Papakyriakopoulos, On Dehn's lemma and the asphericity of knots, Ann. of Math. (2) 66 (1957), 1-26. MR 19, 761.

91. — On solid tori, Proc. London Math. Soc. (3) 7 (1957), 281-299. MR 19, 441.

92. A. Schoenflies, Die Entwicklung der Lehre von den Punktmannigfaltigkeiten. Vol. II, Teubner, Leipzig, 1908.

93. C. L. Seebeck III, Tame arcs on wild cells, Proc. Amer. Math. Soc. 29 (1971), 197-201. MR 43 \#6896.

94. A. Shapiro and J. H. C. Whitehead, A proof and extension of Dehn's lemma, Bull. Amer. Math. Soc. 64 (1958), 174-178. MR 21 \#2242.

95. R. B. Sher, Tame polyhedra in wild cells and spheres, Proc. Amer. Math. Soc. 30 (1971), 169-174. MR 43 \#6897.

96. A. C. Shilepsky, Homogeneity by isotopy for simple closed curves, Duke Math. J. 40 (1973), 463-472. MR 47 \#7725. 
97. A. C. Shilepsky, Homogeneity and extension properties of embeddings of $S^{1}$ in $E^{3}$, Trans. Amer. Math. Soc. 195 (1974), 265-276.

98. J. R. Stallings, Uncountably many wild disks, Ann. of Math. (2) 71 (1960), 185-186. MR 22 \#1871.

99. - On the loop theorem, Ann. of Math. (2) 72 (1960), 12-19. MR 22 \#12526.

100. F. Waldhausen, Eine Verallgemeinerung des Schleifensatzes, Topology 6 (1967), 501504. MR 36 \#3366.

101. J. H. C. Whitehead, On 2-spheres in 3-manifolds, Bull. Amer. Math. Soc. 64 (1958), 161-166. MR 21 \#2241.

102. G. T. Whyburn, On sequences and limiting sets, Fund. Math. 25 (1935), 408-426.

103. - Topological characterization of the Sierpinski curve, Fund. Math. 45 (1958), 320-324, MR 20 \#6077.

104. R. L. Wilder, Partially free subsets of euclidean n-space, Michigan Math. J. 9 (1962), 97-107. MR 25 \#3510.

Department of Mathematics, University of Utah, Salt Lake City, Utah 84112 